\title{
Superconductivity in intercalated group-IV honeycomb structures
}

\author{
José A. Flores-Livas ${ }^{1}$ and Antonio Sanna ${ }^{1}$ \\ ${ }^{1}$ Max-Planck Institut für Microstrukture Physics, Weinberg 2, 06120 Halle, Germany
}

(Dated: May 29, 2022)

\begin{abstract}
We present a theoretical investigation on electron-phonon superconductivity of honeycomb $\mathrm{MX}_{2}$ layered structures. Where $\mathrm{X}$ is one element of the group-IV (C, Si or $\mathrm{Ge}$ ) and $\mathrm{M}$ an alkali or an alkaline-earth metal. Among the studied composition we predict a $\mathrm{T}_{\mathrm{C}}$ of $7 \mathrm{~K}$ in $\mathrm{RbGe}_{2}, 9 \mathrm{~K}$ in $\mathrm{RbSi}_{2}$ and $11 \mathrm{~K}$ in $\mathrm{SrC}_{2}$. All these compounds feature a strongly anisotropic superconducting gap. Our results show that despite the different doping level and structural properties, the three families of materials fall into a similar description of its superconducting behavior. This allows us to estimate an upper critical temperature of about $20 \mathrm{~K}$ for the class of intercalated group-IV structures, including intercalated graphite and doped graphene.
\end{abstract}

PACS numbers:

A large research effort has been lately focused on atomic-thin layered materials and their properties $^{1-3}$. This was triggered by the creation of graphene from graphite $^{4}$ and also motivated by the belief in many potential applications since thin systems can be significantly modified in their electronic properties simply by acting on parameters as stacking, chemical and physical doping ${ }^{5,6}$. In fact this versatility is an extraordinary playground for searching for new superconductors $(\mathrm{SC})^{7}$. Many (low temperature) SC are already known in the class of graphite intercalated compounds $\mathrm{GICs}^{8-12}$, graphene itself has been predicted to superconduct with a critical temperature $\left(\mathrm{T}_{\mathrm{C}}\right)$ of $18 \mathrm{~K}$ upon Li doping ${ }^{13}$.

Among all possible compounds, those chemically and structurally closer to graphite are the honeycomb lattices of silicon ${ }^{14-17}$ and germanium ${ }^{18,19}$. For which suplerconductivity upon intercalation was also reported ${ }^{14,20-24}$. Hence GICs and doped graphene are not unique systems, having a Si and Ge counterpart can be seen as members of a generalized family of group-IV intercalated honeycomb lattices (gIV-ICs).

So far the highest $\mathrm{T}_{\mathrm{C}}$ reported on gIV-ICs is $11.5 \mathrm{~K}$ in $\mathrm{CaC}_{6}{ }^{11,12}$. This system is also the most studied among the family and its superconducting properties are rather well understood ${ }^{6,25-28}$. It is particularly clear that an important role is played by the existence at the Fermi level of 2D electron like bands as well as anti-bonding $\mathrm{C}-\pi$ states. It is also know that a sufficiently large intercalation is therefore a necessary condition to obtain high critical temperatures. But what is the highest conceivable $\mathrm{T}_{\mathrm{C}}$ in an intercalated graphite-like system? Could $\mathrm{Si}$ and Ge iso-morphs be better candidates than GICs? We will address these questions by focusing our investigation on the high doping limit, with one intercalating atom per two honeycomb atoms. We will indicate this family of compounds as $\mathrm{MX}_{2}$ where $\mathrm{M}$ stands for a metal of the $\mathrm{I}$ and II column of the periodic table and $\mathrm{X}$ is carbon, silicon or germanium. This composition is known to occur ${ }^{23}$ in several silicides ${ }^{15,21,22}$ and germanides ${ }^{18,19,24}$.

We will show by means of theoretical ab-initio methods, that finding high temperature superconductivity in these families is a false hope. On the other hand break- ing the record critical temperature of $\mathrm{CaC}_{6}$ is likely to be possible.

All systems are structurally relaxed within Kohn-Sham density-functional theory. ${ }^{29}$ Upon relaxation ${ }^{30}$ all carbon compounds, apart from $\mathrm{CaC}_{2}$, converged to the $\mathrm{AlB}_{2}$ crystal structure (space group $P 6 / \mathrm{mmm}$, number 191), while all silicides and germanides as well as $\mathrm{CaC}_{2}$ converged to the $\mathrm{EuGe}_{2}$ crystal structure (space group $P \overline{3} m 1$, number 164). In both, M occupies the $1 a$ Wyckoff position $(0,0,0)$ and $\mathrm{X}$ the the $2 d$ positions $(1 / 3,2 / 3$, $z)$ and $(2 / 3,1 / 3,-z)$. In the $\mathrm{AlB}_{2}$ structural prototype the parameter $z$ is fix to $1 / 2$, while in the $\mathrm{EuGe}_{2}$ structure it is related to a buckling $(\beta)$ of the honeycomb lattice: $\beta=(|z-1 / 2| \cdot c)$. The $\mathrm{EuGe}_{2}$ structural prototype and the values of $\beta$ are shown in Fig. 1. This figure shows clearly that intercalating lighter ions $(\mathrm{Li}, \mathrm{Be}$, $\mathrm{Na}$ ) induce high buckled honeycomb plans, while heavier ions $(\mathrm{Rb}, \mathrm{Cs}, \mathrm{Ba})$ tend to induce low-buckled plans. $\mathrm{CaC}_{2}$ deviates from the general trend, this structure has a mixture of $s p^{2}-s p^{3}(75 \%-25 \%$ respectively) bonding and therefore at ambient pressure it present a finite buckling (energetically more favorable than in a flat $\mathrm{AlB}_{2}$ structure). In this respect, it has been recently predicted by $\mathrm{Li}$ and coworkers ${ }^{31}$ that the flat-layered phase could be stabilized at high-pressures.

As many of the compounds discussed in this work are not experimentally known, in order to assert on their potential synthesis we calculated their thermodynamic stability, this is derived from the total DFT energy of the system $\left(\mathrm{MX}_{2}\right)$ and of its elemental ground state solid (see supplemental material ${ }^{30}$ for details). This analysis leads to the conclusion that all graphite compounds in the $\mathrm{MX}_{2}$ layered phase are unstable towards this elemental decomposition. While most of silicides and germanides are stable towards decomposition. Nevertheless, since a positive formation energy does not completely exclude these materials from their possible synthesis, we will also investigate their dynamical stability (phonons).

For all systems under investigation we computed phonons and only for those systems dynamically stable, the electron-phonon coupling was calculated by means of density-functional perturbation theory. ${ }^{32}$ We found most 


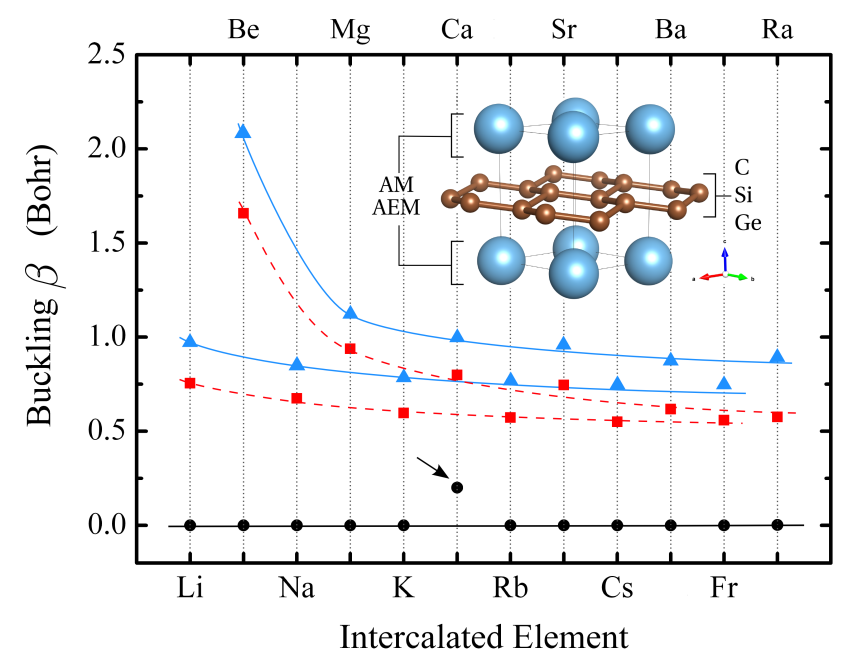

Figure 1. (Color online) Buckling $(\beta)$ of the honeycomb layer as a function of the chemical composition from theoretical structural relaxation. Intercalated graphites are shown as black dots, silicides as red squares and germanides as blue triangles. Lines are guide to the eye to stress the different behavior of alkali and alkaline earth intercalation. The inset shows a prototype crystal structure in a buckled configuration $\left(\beta \neq 0, \mathrm{EuGe}_{2}\right.$ crystal type).

of the intercalated carbon compounds to be dynamically unstable, with the only exception of $\mathrm{Sr}$ and $\mathrm{Ca}$ intercalation. This suggests that the 1 to 2 intercalation is too large for this family and is evidenced experimentally by the reported challenging synthesis of $\mathrm{LiC}_{2}{ }^{8}$, that turns out to be metastable, partially loosing its Li content and converting in $\mathrm{LiC}_{6}{ }^{9,10}$. On the other hand, with the exception of light-ion intercalants, most of the disilicides and digermanides are dynamically stable.

Eliashberg spectral functions ${ }^{33,34} \alpha^{2} F(\omega)$ for all the dynamically stable systems are reported in Fig. 2. From now on we will only consider this subset of materials. In this figure we can clearly observe that the spectral functions are scaled in their frequency by the mass of the atom forming the honeycomb layer. And this extends not only, obviously, to the high energy modes that originate from strong in-layer bonds, but also to the low frequency modes that are dominated by the intercalant motion in the weak interlayer potential. Thus, indicating a chemical effect. We also observe that alkali metals (as compared with alkaline earths) lead to systematically lower phonon branches, therefore to an enhanced coupling strengths ${ }^{33}$

$$
\lambda=2 \int \frac{\alpha^{2} F(\omega)}{\omega} d \omega
$$

at the same time this lowers the average frequency, that we conventionally express as

$$
\omega_{\log }=\exp \left[\frac{2}{\lambda} \int \alpha^{2} F(\omega) \frac{\ln (\omega)}{\omega} d \omega\right]
$$

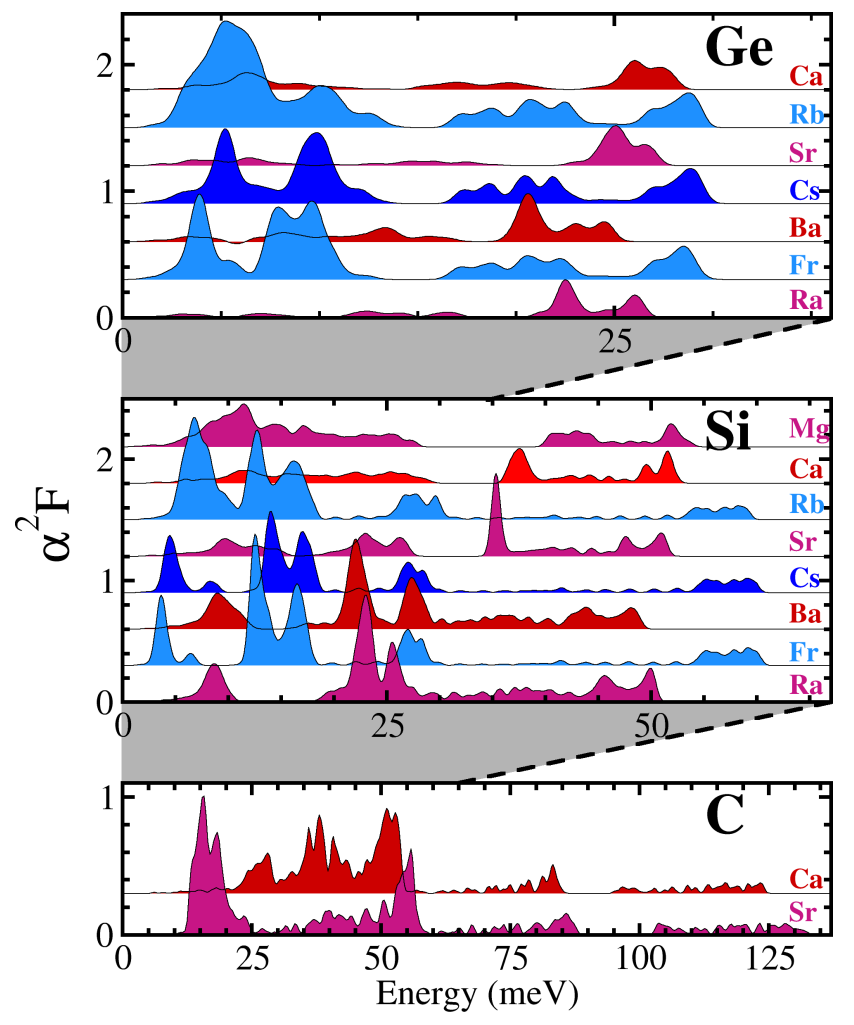

Figure 2. (Color online) Eliashberg spectral function $\alpha^{2} F(\omega)$ calculated for dynamically stable materials on the $\mathrm{MX}_{2}$ set under investigation. These spectral functions show an overall similar behavior, having: a low energy region dominated by intercalant phonon modes, a middle energy range with outof-plane $X$ phonon modes and an high energy spectra of $X$ bonds stretching modes. These features are also seen in $\mathrm{CaC}_{6}$ and doped graphene ${ }^{13,25}$. Note that the plots have different frequency scale, as the mass of the honeycomb atoms scales the entire spectral function and for both: low and high energy regions.

From an electronic point of view, all the materials share a qualitative similar structure. As in the case of $\mathrm{CaC}_{6}$ or doped graphene, there are two type of electronic states located at the Fermi energy: anti-bonding $\pi$ states provided by the honeycomb layer $(\mathrm{C}, \mathrm{Si}, \mathrm{Ge})$ and $2 \mathrm{D}$ interlayer states with contributions from the $\mathrm{M} d$-orbitals. These electronic states hybridize differently along the alkali or the alkaline-earth column and lead to different effective doping and band alignment. This affects the density of states at the Fermi energy $\left(N\left(E_{\mathrm{F}}\right)\right)$ and whit it the occurrence of superconductivity, as we will show below.

In order to perform a fast screen of our $\mathrm{MX}_{2}$ set, the superconducting critical temperatures were estimated within McMillan-Allen-Dynes parametrization of the Eliashberg equations ${ }^{33,35-37}$

$$
T_{\mathrm{c}}=\frac{\omega_{\mathrm{log}}}{1.2 k_{B}} \exp \left[-\frac{1.04(1+\lambda)}{\lambda-\mu^{*}(1+0.62 \lambda)}\right]
$$


where $k_{B}$ is the Boltzmann constant. This formula depends on three parameters: the Coulomb pseudopotential $\mu^{*}$ (here fixed to 0.1 by comparison with SCDFT results, see below); the logarithmic average of the phonon frequency $\omega_{\text {log }}$; and the coupling constant $\lambda$. The computed $\mathrm{T}_{\mathrm{C}}$ couplings $\lambda$ and $\omega_{\text {log }}$ are shown in Fig. 3.

In the limit of an homogeneous coupling in $\mathbf{k}$-space, $\lambda$ is proportional to $N\left(E_{\mathrm{F}}\right)$. Within BCS theory, this parameter splits as $\lambda=V N\left(E_{\mathrm{F}}\right)$, where $V$ is the BCS coupling strength. In Fig. $3 \mathrm{~b}$ ) we observe a remarkable proportionality between $\lambda$ and $N\left(E_{\mathrm{F}}\right)$. Leading to the conclusion that $V$ is approximately the same on this $\mathrm{MX}_{2}$ class of systems, with the sole exception of few systems characterized by strong softening. Eventually this softening will leads to a phononic instability and to a structural phase transition. Perhaps under different thermodynamic conditions of pressure and temperature. Although not belonging to this $\mathrm{MX}_{2}$ family we observe that $\mathrm{CaC}_{6}$ lies perfectly in this regime ${ }^{28,38}$. And similarly does $\mathrm{MgB}_{2}$, however, this is accidental as we have ignored its multi-band nature ${ }^{39-41}$.

These calculations predict several interesting superconductors and in particular $\mathrm{RbSi}_{2}, \mathrm{RbGe}_{2}$ and $\mathrm{SrC}_{2}$. $\mathrm{RbGe}_{2}$ has the highest density of states and, as discussed above, also presents the highest $\lambda$, even though it shows a modest $\mathrm{T}_{\mathrm{C}}$ of $7 \mathrm{~K}$. In fact $\mathrm{T}_{\mathrm{C}}$ (see Eq. 3) depends also on the phonon energy, which is larger for systems having lower mass, for instance $\mathrm{SrC}_{2}$. Also in this Fig. 3 (in panel a), we included the iso-mass lines as a reference to indicate how the $\mathrm{T}_{\mathrm{C}}$ in a material would be affected by $\lambda$ (on $X_{2}$ ) or by $N\left(E_{\mathrm{F}}\right)$. The outcome of this analysis suggests the existence of an upper critical temperature for each family. And this is imposed by the electronic structure, as $N\left(E_{\mathrm{F}}\right)$ hardly would exceed the value of 0.7 states $/ \mathrm{eV} / \mathrm{spin}$ (of $\mathrm{RbGe}_{2}$ ). Following the iso-mass lines in this figure for each subfamily, leads to the conclusion that an upper critical temperature of about $10 \mathrm{~K}$, $15 \mathrm{~K}$ and $20 \mathrm{~K}$ exists respectively for intercalations in $\mathrm{Ge}$, $\mathrm{Si}$ and carbon honeycombs. We firmly believe that this conclusion can be extended beyond the $\mathrm{MX}_{2}$ class, since different intercalation density will not plausibly affect the coupling strength. However, the coupling strength could be significantly affected if $\sigma$ states were involved (as in $\mathrm{MgB}_{2}$ ), but this would require an unphysical doping level.

We will now focus our investigation on three selected systems $\mathrm{SrC}_{2}, \mathrm{RbSi}_{2}$ and $\mathrm{RbGe}_{2}$ as the most interesting representative of each sub-family. As discussed in the introduction both $\mathrm{RbSi}_{2}$ and $\mathrm{RbGe}_{2}$ are stable towards elemental decomposition. In addition they are also more stable than their $\mathrm{RbSi}_{6}$ and $\mathrm{RbGe}_{6}$ rhombohedral counterparts $^{30}$. Therefore, we believe, these two systems are likely to be accessible to the experimental synthesis. On the other hand, $\mathrm{SrC}_{2}$ is not stable with respect to elemental decomposition and turn to be less energetically competitive than its rhombohedral $\mathrm{SrC}_{6}$ configuration that, in fact, has been synthesized ${ }^{42}$. Nevertheless, since the system is dynamically stable, it may still be possi-
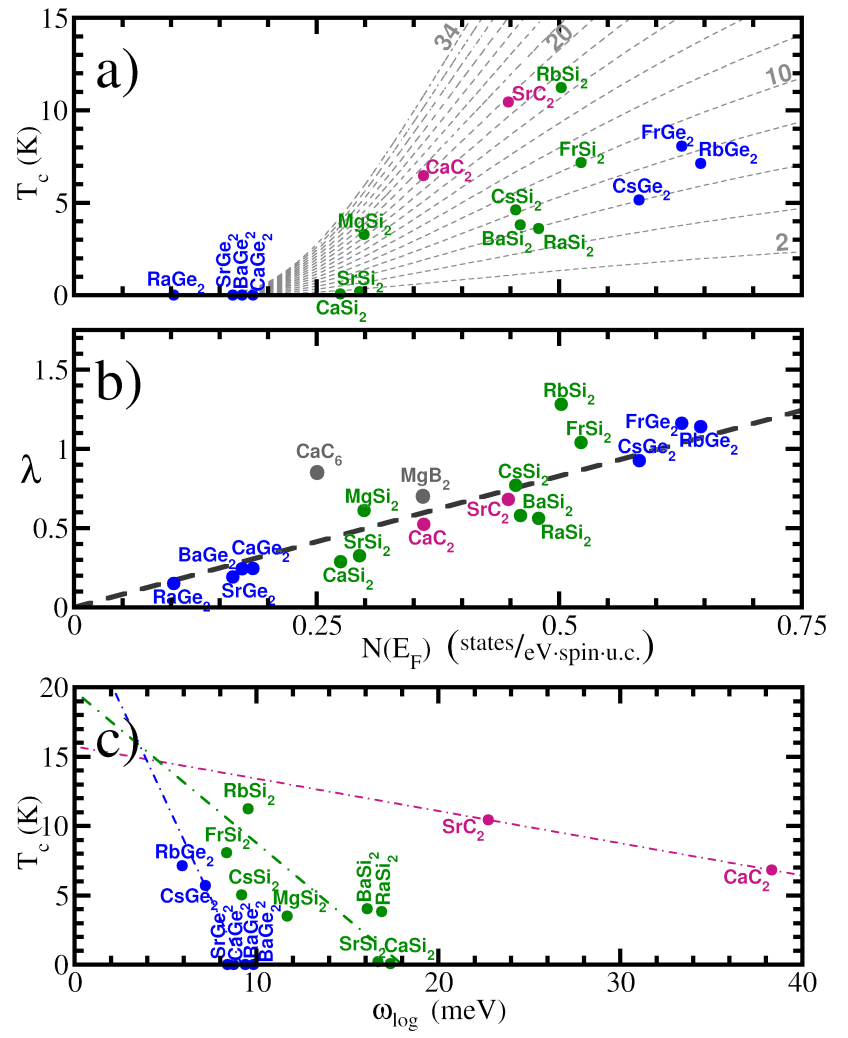

Figure 3. (Color online) a) Critical temperatures calculated with within the McMillan-Allen Dynes formula ${ }^{35,36}$ using $\mu^{*}=0.1$. Grey dashed lines are isomass lines representing the phonon energy average $\omega_{\text {log }}$ (Eq. 2) ranging from 2 to $34 \mathrm{meV}$; b) Electron-phonon coupling parameter $\lambda$ (Eq. 1) as a function of the density of state at the Fermi level $\left(N\left(E_{F}\right)\right)$; c) Critical temperature versus $\omega_{\text {log }}$ (lines serves only as guide).

ble to find a way to its synthesis, perhaps by means of a non-equilibrium process or by high temperature and high pressure, as often used to synthesize clathrates, ${ }^{43}$ carbon borides, ${ }^{44}$ and layered disilicides ${ }^{15,17,21,22}$ and germanides $^{18}$.

The electronic band structures of these three selected materials are shown in Fig. 4. The bands of $\mathrm{SrC}_{2}$ essentially differ from those of $\mathrm{RbSi}_{2}$ and $\mathrm{RbGe}_{2}$, due to the effect of symmetry breaking (both have buckling) and as well the doping level of the honeycomb lattice (charge projection shows that divalent strontium donates 1.2 electrons - while monovalent $\mathrm{Rb}$ donates 0.5 electrons for both $\mathrm{RbSi}_{2}$ and $\mathrm{RbGe}_{2}$ ). The Fermi surfaces (FS) shown in Fig. 5 a, b and c, present multiple Fermi sheets with different orbital character. In $\mathrm{SrC}_{2}$ the inner FS comes from interlayer states, while the outer surface is formed by carbon $\pi$ states. In $\mathrm{RbSi}_{2}$ and $\mathrm{RbGe}_{2}$ the hybridization between interlayer and honeycomb $\pi$ states is much stronger. The outer FS is mostly due to $\mathrm{Si} / \mathrm{Ge}$ $\pi$ states, while the inner FS has an interlayer character, however with a relatively large overlap (25\%) to $\mathrm{Si} / \mathrm{Ge} \pi$ states.

The phonon dispersion for the three systems is shown 


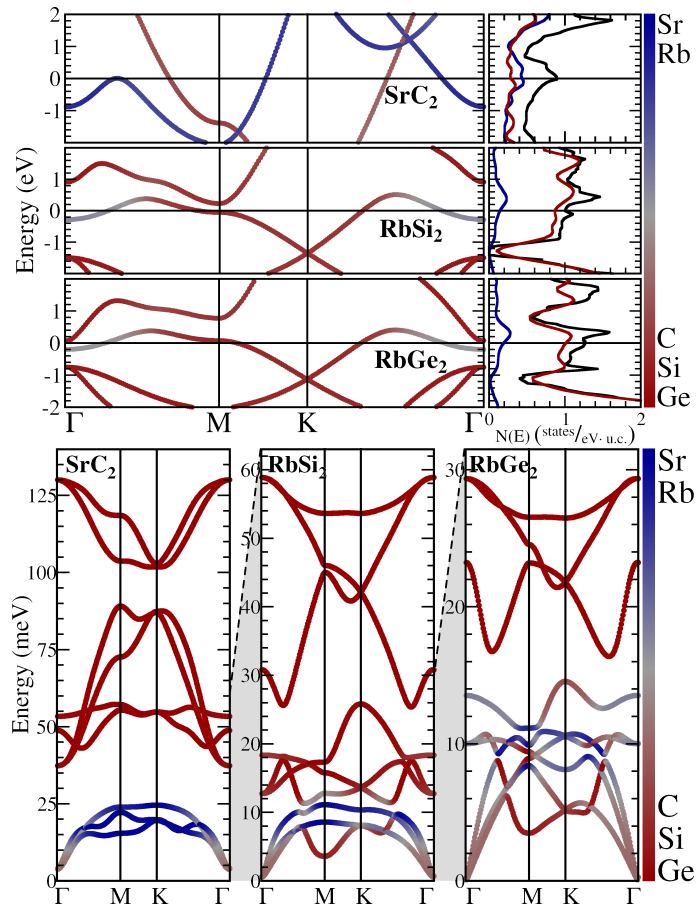

Figure 4. (Color online) Top: Electronic bands in $\mathrm{SrC}_{2}, \mathrm{RbSi}_{2}$ and $\mathrm{RbGe}_{2}$ around the Fermi level (at zero eV). The color scale indicates the projection of the Kohn-Sham states on the atomic orbitals of the intercalating atom. Bottom. Phonon dispersion relation. The color scale indicates the component of the phonon mode on the intercalant atom.

in Fig. 4. The overall structure of the phonon modes is the same for the three systems. Low frequency modes present a strong intercalant component, fundamentally due to the weak force constants that binds the $M$ atoms to their position in the lattice, but also because of their relatively large mass. To the scope of this work, the most interesting feature of the phononic dispersion is the behavior of the buckling modes. In the unbuckled (flat) $\mathrm{SrC}_{2}$ compound this mode has $50 \mathrm{meV}$ in the zone center and and cannot falls below $40 \mathrm{meV}$. While in the buckled $\mathrm{RbSi}_{2}$ and $\mathrm{RbGe}_{2}$ compounds it becomes "soft" moving from $\Gamma$ ( at $30 \mathrm{meV}$ in $\mathrm{RbSi}_{2}$ and $23 \mathrm{meV}$ in $\mathrm{RbGe}_{2}$ ) to $M$ $(3.5 \mathrm{meV})$. This mode is strongly coupled in both $\mathrm{RbSi}_{2}$ and $\mathrm{RbGe}_{2}$, and anharmonic effects (not considered in the present work) may also affect the strength of its coupling.

We will now reconsider the superconducting properties of these selected systems by means of a more accurate superconductivity theory than the McMillan formula used so far. We will adopt density-functional theory for superconductors (SCDFT), as it is completely parameter free $^{45-47}$ and allows for a full k-resolved description ${ }^{48}$

It should be observed (see Fig. 5 on panels a, b and c) that the electron phonon coupling in all these systems is rather anisotropic, meaning strongly $\mathbf{k}$-dependent on

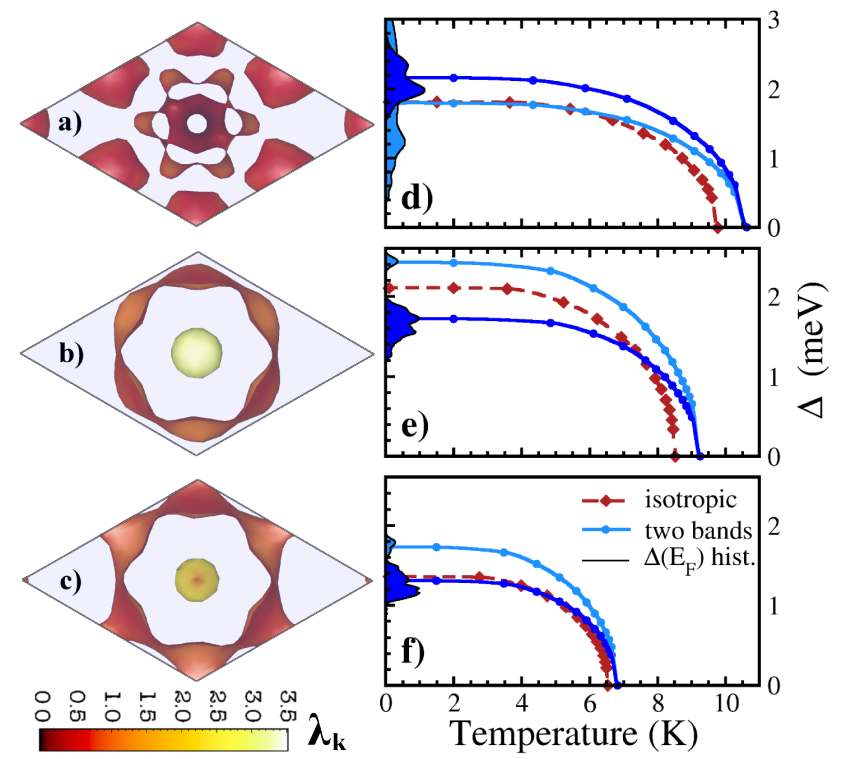

Figure 5. (Color online) Fermi surface of $\mathrm{SrC}_{2}$ (a) $\mathrm{RbSi}_{2}$ (b) and $\mathrm{RbGe}_{2}$ (c), shown in the $\Gamma$ centered reciprocal unit cell (top view). The color scale (bottom left corner) gives the k-resolved electron phonon coupling ${ }^{28} \lambda_{k}$. Superconducting gap as a function of temperature for $\mathrm{SrC}_{2}$ (d) $\mathrm{RbSi}_{2}$ (e) and $\mathrm{RbGe}_{2}$ (f), computed within SCDFT ${ }^{49}$. The red-dashed line is the isotropic behavior, blue-continuous lines are a minimal two-band approximation. The full gap distribution func$\operatorname{tion}^{28,38}$ is given at $T=0$ in as a filled area.

the FS. $\mathrm{SrC}_{2}$ has a continuous distribution, while the two FS of $\mathrm{RbSi}_{2}$ and $\mathrm{RbGe}_{2}$, have remarkably different coupling strength: stronger on the small FS around the $\Gamma$ point and weaker in the outer FS (at large $|\mathbf{k}|$ ). The distribution of superconducting gaps on the Fermi energy (not shown) follows the anisotropy in $\lambda_{\mathbf{k}}$, similarly to the behavior observed in bulk lead ${ }^{50}$. The gap distribution function at $T=0$ (i.e. the energy distribution of the SC gaps: $\Delta_{k_{F}}$ ), as well as the temperature dependence (in a two-band and single band model) are plotted in Fig. 5 d,e,f. Both $\mathrm{RbSi}_{2}$ and $\mathrm{RbGe}_{2}$ show two distinct gaps (like in $\mathrm{MgB}_{2}$ or bulk lead ${ }^{40,41,50}$ ), while $\mathrm{SrC}_{2}$ has an anisotropic gap continuously distributed. This gap distribution reminds that of $\mathrm{CaC}_{6}{ }^{28,51,52}$. This anisotropy will affect the specific heat and the thermodynamical properties. However, unlike in $\mathrm{MgB}_{2}$, the critical temperature is not much affected by it (less than $1 \mathrm{~K}$ ). The role of coupling anisotropy on the superconducting behavior can be clearly understood within the qualitative model of Suhl, Mattias and Walker ${ }^{53}$. The observed combination of a large anisotropy in the gap with a small enhancement in $\mathrm{T}_{\mathrm{C}}$ is a consequence of the strong inter-band coupling between $\pi$-states (having a smaller gap) and the interlayer states (that dominate on the larger gap). The gap distribution of $\mathrm{SrC}_{2}$ is even broader and clearly cannot be completely captured within a two-band model. The system is in fact almost gapless, since the small $|\mathbf{k}|$ part of the FS shows a negligible superconducting pairing as 
a consequence of the weak phononic coupling.

In summary, we presented a theoretical study on honeycomb layered binary carbides, silicides and germanides intercalated by alkali or alkaline-earth metals. Our superconductivity analysis has shown that in this class of materials are many compounds with a relatively high critical temperature $(\sim 10 \mathrm{~K})$ as well as a quite complex superconducting state. In addition, the stability investigation has shown that several compounds should be accessible to their experimental synthesis. Finally, we demonstrate an intrinsic physical similarity among the group, which can be traced back to their characteristic $\pi+$ interlayer character of states at the Fermi surface. From this feature we estimate an upper limit for the transition critical temperatures: $\sim 20 \mathrm{~K}, \sim 15 \mathrm{~K}$ and $\sim 10 \mathrm{~K}$ respectively for carbon, silicon and germanium intercalated honeycombs. This limit could be broken only in the unlikely case in which the doping level would be able to drive $\sigma$-states at the Fermi level. Nevertheless this study indicates that superconductivity in doped graphite and similar systems is a rather general behavior and many more superconductors may still be discovered.

J.A.F.L. acknowledge financial support from EU's 7th Framework Marie-Curie scholarship Program within the "ExMaMa" Project (329386).
${ }^{1}$ a. K. Geim and K. S. Novoselov, Nat. Mat. 6, 183 (2007).

2 a. K. Geim, Science 324, 1530 (2009).

${ }^{3}$ K. S. Novoselov, V. I. Fal'ko, L. Colombo, P. R. Gellert, M. G. Schwab, and K. Kim, Nature 490, 192 (2012).

${ }^{4}$ K. S. Novoselov, A. K. Geim, S. V. Morozov, D. Jiang, Y. Zhang, S. V. Dubonos, I. V. Grigorieva, and A. A. Firsov, Science 306, 666 (2004).

5 A. V. Fedorov, N. I. Verbitskiy, D. Haberer, C. Struzzi, L. Petaccia, D. Usachov, O. Y. Vilkov, D. V. Vyalikh, J. Fink, M. Knupfer, B. Buechner, and A. Grueneis, Nat. Com. 5 (2014).

${ }^{6}$ S.-L. Yang, J. A. Sobota, C. A. Howard, C. J. Pickard, M. Hashimoto, D. H. Lu, S.-K. Mo, P. S. Kirchmann, and Z.-X. Shen, Nat. Com. 5 (2014).

7 R. A. Klemm, Book published by Oxford University Press, USA (2012), book.

8 I. Belash, A. Bronnikov, O. Zharikov, and A. Pal'nichenko, Solid State Comm. 69, 921 (1989).

9 V. A. Nalimova, D. Guerard, M. Lelaurain, and O. V. Fateev, Carbon 33, 177 (1995).

10 V. V. Avdeeva, V. A. Nalimovaa, and K. N. Semenenko, High Press. Res. 6, 11 (1990).

11 T. E. Weller, M. Ellerby, S. S. Saxena, R. P. Smith, and N. T. Skipper, Nat. Phys. 1 (2005).

12 N. Emery, C. Hérold, M. d'Astuto, V. Garcia, C. Bellin, J. F. Marêché, P. Lagrange, and G. Loupias, Phys. Rev. Lett. 95, 087003 (2005).

13 G. Profeta, M. Calandra, and F. Mauri, Nature Phys. 8, 131 (2012).

14 S. Sanfilippo, H. Elsinger, M. Nunez-Regueiro, O. Laborde, S. LeFloch, M. Affronte, G. L. Olcese, and A. Palenzona, Phys. Rev. B 61, R3800 (2000).

15 P. Bordet, M. Affronte, S. Sanfilippo, M. Nunez-Regueiro, O. Laborde, G. L. Olcese, A. Palenzona, S. LeFloch, D. Levi, and M. Hanfland, Phys. Rev. B 62, 11392 (2000).

16 M. Imai, T. Hirano, T. Kikegawa, and O. Shimomura, Phys. Rev. B 58, 11922 (1998).

17 M. Imai and T. Kikegawa, Chem. Mater. 15, 2543 (2003).

18 P. H. Tobash and S. Bobev, Journal of Solid State Chemistry 180, 1575 (2007).

19 S. Bobev, E. D. Bauer, J. D. Thompson, J. L. Sarrao, G. J. Miller, B. Eck, and R. Dronskowski, Journal of Solid State Chemistry 177, 3545 (2004).

${ }^{20}$ K. H. M. Imai and T. Hirano, Physica C 245C, 12 (1995).

21 J. A. Flores-Livas, R. Debord, S. Botti, A. San Miguel,
M. A. L. Marques, and S. Pailhès, Phys. Rev. Lett. 106, 087002 (2011).

22 J. Evers and A. Weiss, Mater. Res. Bull. 9, 549 (1974).

23 R. Demchyna, S. Leoni, H. Rosner, and U. Schwarz, Z. Kristallogr. 221, 420 (2006).

24 S. Yamanaka, Dalton Trans. 39, 1901 (2009).

25 M. Calandra and F. Mauri, Phys. Rev. Lett. 95, 237002 (2005).

${ }^{26}$ L. Boeri, G. B. Bachelet, M. Giantomassi, and O. K. Andersen, Phys. Rev. B 76, 064510 (2007).

27 J. S. Kim, L. Boeri, R. K. Kremer, and F. S. Razavi, Phys. Rev. B 74, 214513 (2006).

28 A. Sanna, G. Profeta, A. Floris, A. Marini, E. K. U. Gross, and S. Massidda, Phys. Rev. B 75, 020511 (2007).

${ }^{29}$ We used the two plane-wave based code ABINIT $^{54}$, and ESPRESSO $^{55}$ within the Perdew-Burke-Ernzerhof $(\mathrm{PBE})^{56}$ exchange correlation functional and the core states were accounted for by norm-conserving Troullier-Martins pseudopotentials $^{57}$. The pseudopotential accuracy has been checked against all-electron (LAPW+lo) method as implemented in the ELK code (http://elk.sourceforge.net/).

30 Supplemental Material.

31 Y.-L. Li, W. Luo, Z. Zeng, H.-Q. Lin, H.-k. Mao, and R. Ahuja, Proc. Nat. Ame. Soc. 110 (2013).

32 The phonon spectrum and the electron-phonon matrix elements were obtained employing density-functional perturbation theory. ${ }^{58-60}$, within the pseudopotential approximation. A cutoff energy of $60 \mathrm{Ry}$ was used in the plane-wave expansion. A $16 \times 16 \times 12$ Monkhorst-Pack $k$-grid and a $4 \times 4 \times 2 q$-grid was used for all the materials under consideration. With the only exception of $\mathrm{SrC}_{2}, \mathrm{RbSi}_{2}$ and $\mathrm{RbGe}_{2}$, where we have increased the $q$ sampling grid to $8 \times 8 \times 6$ in order to achieve an accurate description of anisotropic properties.

33 J. P. Carbotte, Rev. Mod. Phys. 62, 1027 (1990).

${ }^{34}$ P. B. Allen and B. Mitrović (Academic Press, 1983) pp. 1 $-92$.

${ }^{35}$ W. L. McMillan, Phys. Rev. 167, 331 (1968).

36 P. B. Allen and R. C. Dynes, Phys. Rev. B 12, 905 (1975).

37 G. Eliashberg, Teor. Fiz 38, 966 (1960)[Sov. Phys. FETP 38 (1960).

38 A. Sanna, S. Pittalis, J. K. Dewhurst, M. Monni, S. Sharma, G. Ummarino, S. Massidda, and E. K. U. Gross, Phys. Rev. B 85, 184514 (2012).

39 A. Y. Liu, I. I. Mazin, and J. Kortus, Phys. Rev. Lett. 87, 
087005 (2001).

40 A. Floris, G. Profeta, N. N. Lathiotakis, M. Lüders, M. A. L. Marques, C. Franchini, E. K. U. Gross, A. Continenza, and S. Massidda, Phys. Rev. Lett. 94, 037004 (2005).

41 A. Floris, A. Sanna, M. Lüders, G. Profeta, N. Lathiotakis, M. Marques, C. Franchini, E. Gross, A. Continenza, and S. Massidda, Physica C: Superconductivity 456, 45 (2007).

42 J. S. Kim, L. Boeri, J. R. O'Brien, F. S. Razavi, and R. K. Kremer, Phys. Rev. Lett. 99, 027001 (2007).

43 P. Toulemonde, C. Adessi, X. Blase, A. San Miguel, and J. L. Tholence, Phys. Rev. B 71, 094504 (2005).

44 S. Shah and A. N. Kolmogorov, Phys. Rev. B 88, 014107 (2013).

${ }^{45}$ L. N. Oliveira, E. K. U. Gross, and W. Kohn, Phys. Rev. Lett. 60, 2430 (1988).

46 M. Lüders, M. A. L. Marques, N. N. Lathiotakis, A. Floris, G. Profeta, L. Fast, A. Continenza, S. Massidda, and E. K. U. Gross, Phys. Rev. B 72, 024545 (2005).

47 M. A. L. Marques, M. Lüders, N. N. Lathiotakis, G. Profeta, A. Floris, L. Fast, A. Continenza, E. K. U. Gross, and S. Massidda, Phys. Rev. B 72, 024546 (2005).

48 The phononic functional we use is an improved version with respect to Ref. 46 and 47 and is discussed in Ref. 49. Coulomb interactions are included within static $\mathrm{RPA}^{28}$.

49 A. Sanna and E. K. U. Gross, (2014), to be published.

50 A. Floris, A. Sanna, S. Massidda, and E. K. U. Gross, Phys. Rev. B 75, 054508 (2007).

51 R. S. Gonnelli, D. Daghero, D. Delaude, M. Tortello, G. A. Ummarino, V. A. Stepanov, J. S. Kim, R. K. Kremer, A. Sanna, G. Profeta, and S. Massidda, Phys. Rev. Lett. 100, 207004 (2008).
${ }^{52}$ U. Nagel, D. Hüvonen, E. Joon, J. S. Kim, R. K. Kremer, and T. Rõ om, Phys. Rev. B 78, 041404 (2008).

${ }^{53}$ H. Suhl, B. Matthias, and L. Walker, Physical Review Letters 3, 552 (1959).

54 X. Gonze, B. Amadon, P. Anglade, J. Beuken, F. Bottin, P. Boulanger, F. Bruneval, D. Caliste, R. Caracas, M. Côté, T. Deutsch, L. Genovese, P. Ghosez, M. Giantomassi, S. Goedecker, D. Hamann, P. Hermet, F. Jollet, G. Jomard, S. Leroux, M. Mancini, S. Mazevet, M. Oliveira, G. Onida, Y. Pouillon, T. Rangel, G. Rignanese, D. Sangalli, R. Shaltaf, M. Torrent, M. Verstraete, G. Zerah, and J. Zwanziger, Computer Physics Communications 180, 2582 (2009).

55 P. Giannozzi, S. Baroni, N. Bonini, M. Calandra, R. Car, C. Cavazzoni, D. Ceresoli, G. L. Chiarotti, M. Cococcioni, I. Dabo, A. Dal Corso, S. de Gironcoli, S. Fabris, G. Fratesi, R. Gebauer, U. Gerstmann, C. Gougoussis, A. Kokalj, M. Lazzeri, L. Martin-Samos, N. Marzari, F. Mauri, R. Mazzarello, S. Paolini, A. Pasquarello, L. Paulatto, C. Sbraccia, S. Scandolo, G. Sclauzero, A. P. Seitsonen, A. Smogunov, P. Umari, and R. M. Wentzcovitch, Journal of Physics: Condensed Matter 21, 395502 (19pp) (2009).

56 J. P. Perdew, K. Burke, and M. Ernzerhof, Phys. Rev. Lett. 77, 3865 (1996).

57 M. Fuchs and M. Scheffler, Comput. Phys. Commun. 119, 67 (1999).

58 S. Baroni, S. de Gironcoli, A. Dal Corso, and P. Giannozzi, Rev. Mod. Phys. 73, 515 (2001).

59 X. Gonze and J.-P. Vigneron, Phys. Rev. B 39, 13120 (1989).

60 S. Y. Savrasov and D. Y. Savrasov, Phys. Rev. B 54, 16487 (1996). 